\title{
Evaporative cooling to a Rydberg crystal close to its ground state
}

\author{
M. Brune $\odot^{1}$ and D. J. Papoular ${ }^{2}$ \\ ${ }^{1}$ Laboratoire Kastler Brossel, Collège de France, CNRS, ENS-Université PSL, Sorbonne Université, France \\ ${ }^{2}$ LPTM, UMR 8089 CNRS \& Université Cergy-Pontoise, France
}

(Received 5 September 2019; accepted 6 March 2020; published 8 April 2020)

\begin{abstract}
We theoretically show how to obtain a long one-dimensional crystal near its quantum ground state. We rely on an evaporative cooling scheme applicable to many-body systems with nonzero-ranged interactions. Despite the absence of periodic potentials, the final state is a crystal that exhibits long-range spatial order. We describe the scheme thermodynamically, applying the truncated Boltzmann distribution to the collective excitations of the chain, and we show that it leads to a novel quasiequilibrium many-body state. For longer chains, comprising about 1000 atoms, we emphasize the quasiuniversality of the evaporation curve. Such exceptionally long one-dimensional (1D) crystals are only accessible deep in the quantum regime. We perform our analysis on the example of an initially thermal chain of circular Rydberg atoms confined to a 1D geometry. Our scheme may be applied to other quantum systems with long-ranged interactions such as polar molecules.
\end{abstract}

DOI: 10.1103/PhysRevResearch.2.023014

\section{INTRODUCTION}

Systems presenting long-ranged interactions exhibit strongly correlated crystalline phases [1-4]. Among them, quantum crystals are those whose constituents undergo large-amplitude zero-point motion [5]. The collective nature of their excitations leads to spectacular phenomena including the Tkachenko oscillations of a vortex lattice in a superfluid [6,7], the giant plasticity of helium crystals [8], and supersolidity in ultracold gases presenting interactions beyond the contact limit [9-13].

Up to now, the investigation of one-dimensional (1D) quantum crystals has been hindered by the difficulty of obtaining large crystals in this geometry, where thermal and quantum fluctuations both destroy long-range order in macroscopic systems [14]. Nevertheless, crystallization does occur in finitesized systems [15]. It has been unambiguously observed in the absence of any external periodic potential only in small systems of up to 50 ions [16-19] or 10 electrons [20,21]. The realization of larger 1D crystals requires going deep into the quantum regime. There, thermal fluctuations are suppressed, and long-range order is only limited by quantum fluctuations, which are less stringent [14]. The realization of large 1D crystals will pave the way toward the investigation of 1D quantum crystals, where one may look for, e.g., giant plasticity through the tunneling of defects $[22,23]$.

We focus on one way of obtaining spatial order that relies on strong nonzero-range dipole interactions between Rydberg atoms [24]. Rydberg atoms are ideally suited for quantum information processing $[25,26]$ and quantum

Published by the American Physical Society under the terms of the Creative Commons Attribution 4.0 International license. Further distribution of this work must maintain attribution to the author(s) and the published article's title, journal citation, and DOI. simulation [27,28]. Nontrivial many-body states [29-31] of up to 50 atoms manipulated with optical tweezers have been prepared through resonant coupling to Rydberg states [32-35]. Rydberg states may be weakly admixed to the atomic ground state [36-38] or resonantly excited [39] so as to study the interplay between anisotropic interactions and disorder or frustration [40]. Quantum gases resonantly coupled to Rydberg states have been predicted to exhibit a quantum phase transition to a Rydberg crystal [41], leading to a universal scaling behavior observed in the critical region [42].

In all those cases, low-angular-momentum Rydberg states were considered, leading to a strong limitation on the lifetime (100 $\mu$ s per atom, a few $\mu$ s for many atoms), limiting the size of the system. Circular Rydberg atoms [43-46], whose excited electron has maximal orbital and magnetic quantum numbers, overcome this limitation and offer a very promising platform for the quantum simulation of many-body problems [27]. Using spontaneous emission inhibition [47,48], their already long lifetime $(30 \mathrm{~ms})$ is expected to be extended to more than $1 \mathrm{~min}$. This timescale allows for implementing an evaporative cooling scheme applicable to Rydberg atoms [27], whose classical analysis shows great promise for reaching extremely low temperatures.

In this article, we show that large 1D Rydberg crystals may be prepared very close to their quantum ground state in realistic experimental conditions [27,49] through this evaporative cooling scheme. Despite the absence of any spatially periodic potential, these crystals exhibit long-range spatial order. This is in stark contrast to the classical analysis of 1D systems, which would predict the absence of long-range order [14]. We introduce a quantum thermodynamic model, applying the truncated Boltzmann distribution to the collective excitations of the chain. We show that it leads to a novel quasiequilibrium regime that differs from the truncated Bose-Einstein distribution applicable to quantum-degenerate gases [50]. In contrast to dilute systems in which the evaporation is driven by two-body collisions [51], the mechanism we describe here 


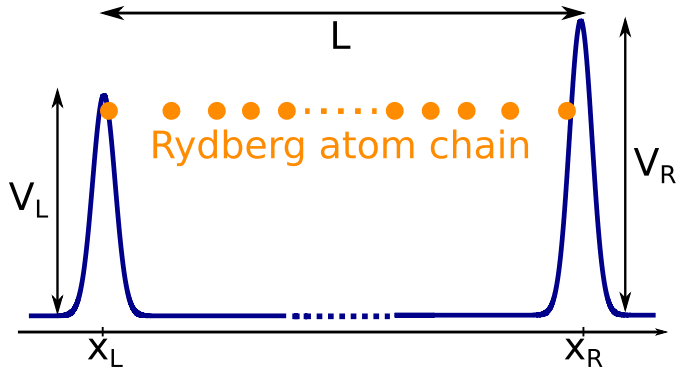

FIG. 1. A Rydberg-atom chain (orange) confined in a 1D trap of size $L$. The potential maxima $V_{L}$ and $V_{R}$ satisfy $V_{L}<V_{R}$, so that atoms are expelled from the left edge of the trap.

hinges on many-body physics, whereby the phonons present in the chain lead to the expulsion of a single atom. Hence, it is related to the quantum evaporation of liquid helium [52-54], also predicted to affect cold bosonic atoms [55].

\section{THE SYSTEM AND THE HAMILTONIAN UNDER CONSIDERATION}

We first consider a fixed number $N$ of Rydberg atoms confined in a 1D trap of fixed size $L$ (see Fig. 1). We illustrate our model using the parameters of Ref. [27]. The atoms are confined radially using the ponderomotive potential [56] induced by a Laguerre-Gaussian laser beam (Ref. [57], Chap. 2). They are trapped axially between two optical plugs yielding the potential $V_{T}(x)=V_{L} \exp \left[-2\left(x-x_{L}\right)^{2} / w^{2}\right]+V_{R} \exp [-2(x-$ $\left.\left.x_{R}\right)^{2} / w^{2}\right]$. The barrier width and heights are, respectively, $w=30 \mu \mathrm{m}, V_{L} / h=3 \mathrm{MHz}$, and $V_{R} / h=4 \mathrm{MHz}$. The trap size $L=x_{R}-x_{L}$ is slowly decreased from its initial value so as to induce successive atomic expulsions, providing the evaporative cooling. Unlike for gases, the barrier heights remain constant during the whole process. The atoms interact via the strongly repulsive van der Waals interaction $V\left(x_{i}, x_{j}\right)=$ $C_{6} /\left|x_{i}-x_{j}\right|^{6}$ with $C_{6} / h=3 \mathrm{GHz} \mu \mathrm{m}^{6}$, corresponding to ${ }^{87} \mathrm{Rb}$ atoms with the principal quantum number $n=50$. The equilibrium positions $x_{1}^{0}, \ldots, x_{N}^{0}$ are evenly spaced in the bulk of the chain, but not on the edges, due to the finite spatial extent of the barriers. Two neighboring atoms are distant by $l \approx$ $5 \mu \mathrm{m}$, leading to interaction energies $C_{6} / l^{6} \approx h \times 200 \mathrm{kHz}$.

We describe the atomic vibrations in terms of a quadratic Hamiltonian:

$$
H=\sum_{k=1}^{N}\left[\frac{\tilde{p}_{k}^{2}}{2 m}+\frac{1}{2} m \omega_{k}^{2} \tilde{u}_{k}^{2}\right] \text { with } \tilde{u}_{k}=\sum_{n=1}^{N} R_{n k} u_{n} .
$$

In Eq. (1), the $N$ vibrational modes $\left\{\tilde{u}_{k}\right\}$ have the frequencies $\omega_{1}<\cdots<\omega_{N}$, and the $\left\{\tilde{p}_{k}\right\}$ are their conjugate momenta. They are related to the atomic displacements $\left\{u_{n}=x_{n}-x_{n}^{0}\right\}$ through the orthogonal matrix $R$. The applicability of Eq. (1) only requires local order (see Appendix A 1): the averages $\left\langle\left(u_{n+1}-u_{n}\right)^{2}\right\rangle^{1 / 2}$ involving two neighboring atoms should remain small compared to $l=L / N$. For a thermal chain at the temperature $T$, this requires $k_{\mathrm{B}} T<2 C_{6} / l^{6}$, and is well satisfied for up to 1000 atoms with $l \sim 5 \mu \mathrm{m}$ and $k_{\mathrm{B}} T \lesssim$ $h \times 100 \mathrm{kHz} \approx k_{\mathrm{B}} \times 5 \mu \mathrm{K}$.

\section{CLASSICAL THERMODYNAMICS}

For a given configuration characterized by the phonon mode energies $\left\{\epsilon_{k}\right\}_{1 \leqslant k \leqslant N}$ and phases $\left\{\phi_{k}\right\}_{1 \leqslant k \leqslant N}$, the position of the leftmost atom at time $t$ is $u_{1}(t)=$ $\sum_{k} R_{1 k}\left[2 \epsilon_{k} /\left(m \omega_{k}^{2}\right)\right]^{1 / 2} \cos \left(\omega_{k} t+\phi_{k}\right)$. It remains trapped as long as $\left|u_{1}(t)\right|<u_{M}$, where $u_{M}=x_{1}^{0}-x_{L}$. We consider the time-averaged mean-square displacement $\left\langle u_{1}^{2}\right\rangle=u_{M}^{2} \sum_{k=1}^{N} \epsilon_{k} / E_{M k}$, where the quantities $E_{M k}=$ $m \omega_{k}^{2} u_{M}^{2} / R_{1 k}^{2}$ increase with $k$. Hence, for a given $\alpha$, the lowest-energy configurations for which $\left\langle u_{1}^{2}\right\rangle^{1 / 2}=\alpha u_{M}$ are those where only the mode $k=1$ is excited, with the energy $E=\epsilon_{1}=\alpha^{2} E_{M 1}$. For $\alpha=1 / \sqrt{2}$, they correspond to atom 1 barely reaching $u_{1}=-u_{M}$, i.e., to the lowest-energy untrapped configurations. Their energy $E_{M}^{\mathrm{cl}}=m \omega_{1}^{2} u_{M}^{2} /\left(2 R_{11}^{2}\right)$ is set by $\omega_{1}$. Furthermore, numerical simulations of the classical (cl) dynamics of the atom chain [58] have shown the atomic motion to be chaotic. Hence, exploiting ergodicity, the trapped configurations are those with $E<E_{M}^{\mathrm{cl}}$. We describe the quasiequilibrium thermodynamics of the chain using a Boltzmann distribution truncated at the energy $E_{M}^{\mathrm{cl}}$, whose partition function reads

$$
Z^{\mathrm{cl}}=\int_{E<E_{M}^{\mathrm{cl}}} \frac{\prod\left[d \tilde{p}_{k} d \tilde{u}_{k}\right]}{h^{N}} e^{-\beta E}=\frac{P\left(N, \beta E_{M}^{\mathrm{cl}}\right)}{\beta^{N} \hbar \omega_{1} \cdots \hbar \omega_{N}} .
$$

In Eq. (2), $\beta=1 /\left(k_{\mathrm{B}} T\right)$ is the inverse temperature, $E=$ $H\left(\left\{\tilde{p}_{k}, \tilde{u}_{k}\right\}\right)$, and $P(a, z)=\gamma(a, z) / \Gamma(N)$ is the normalized lower incomplete gamma function [59]. The mean (quadratic) energy $U^{\mathrm{cl}}(L, T)$ associated with the Hamiltonian $H$ and the entropy $S^{\mathrm{cl}}(L, T)$ follow from $U^{\mathrm{cl}}=-\partial_{\beta} \ln Z^{\mathrm{cl}}$ and $S^{\mathrm{cl}} / k_{\mathrm{B}}=$ $\ln Z^{\mathrm{cl}}-\beta \partial_{\beta} \ln Z^{\mathrm{cl}}$

The function $P(a, z)$ also appears in the thermodynamics of the evaporation of a gas ( $a=3$ for a harmonic trap) [51]. Here, $a=N$ ranges from 40 to 1000 , so that the role of truncation is strongly enhanced with respect to gases of ground-state atoms (see Appendix A 3). It is important for $k_{\mathrm{B}} T \gtrsim E_{M}^{\mathrm{cl}} / N$. For larger $T$, all trapped configurations are equally populated. The probability density for a configuration to have the energy $E$ is $N E^{N-1} /\left(E_{M}^{\mathrm{cl}}\right)^{N}$, hence nearly all configurations have energies $\sim E_{M}^{\mathrm{cl}}$. Both $U^{\mathrm{cl}}$ and $S^{\mathrm{cl}}$ reach finite maxima $U_{\max }^{N}(L)$ and $S_{\max }^{N}(L)$ (see Fig. 2), where

$$
U_{\max }^{(N)}=\frac{N E_{M}^{\mathrm{cl}}}{N+1} \text { and } S_{\max }^{(N)}=k_{\mathrm{B}} \ln \left(\frac{\left(E_{M}^{\mathrm{cl}}\right)^{N} / N !}{\hbar \omega_{1} \cdots \hbar \omega_{N}}\right) .
$$

For fixed $N$, both maxima increase with $L$, because less stringent traps will accommodate higher-energy excitations. This novel regime is inaccessible with gases, where an atom whose energy is close to the evaporation threshold is expelled when it undergoes a collision [51,60]. However, it is accessible for a Rydberg chain (see Appendix A 2).

\section{QUANTUM THERMODYNAMICS}

For lower quadratic energies, we use a quantum (quant) description. Assuming ergodicity in the quantum regime, we introduce the energy $E_{\mathbf{n}}=\sum_{k=1}^{N} \hbar \omega_{k}\left(n_{k}+1 / 2\right)$ of the configuration labeled by the integer multiplet $\mathbf{n}=\left\{n_{k}\right\}_{1 \leqslant k \leqslant N}$. The 


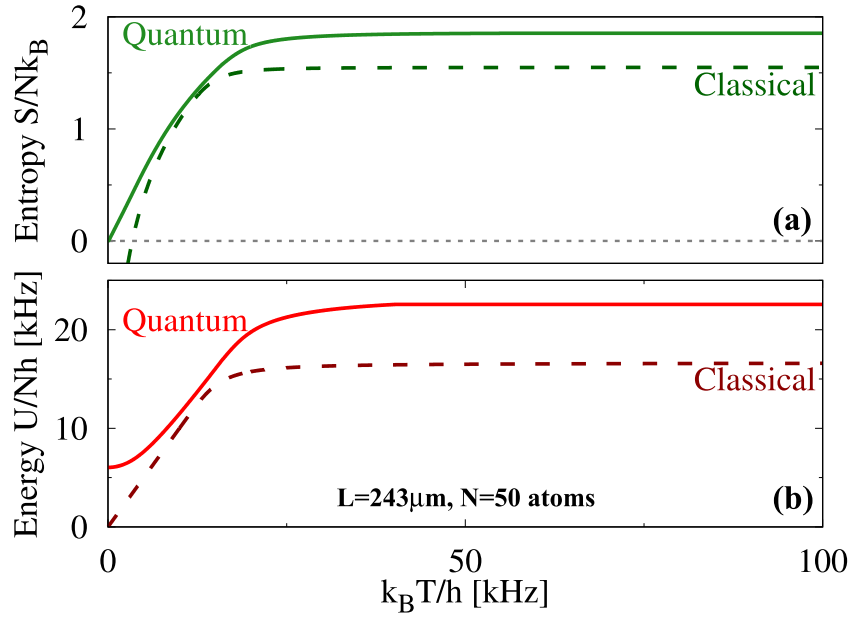

FIG. 2. (a) Entropy and (b) energy per particle for $N=50$ atoms in a trap of size $L=243 \mu \mathrm{m}$ (close to the end of the evaporation for the chain of Fig. 4). The solid (dashed) lines show the quantum (classical) prediction.

threshold energy $E_{M}^{\text {quant }}$ for trapped configurations satisfies

$$
E_{M}^{\text {quant }}=\min _{\mathbf{n}}\left[E_{\mathbf{n}} \text { with } \sum_{k=1}^{N} \frac{\hbar \omega_{k}\left(n_{k}+1 / 2\right)}{E_{M k}} \geqslant \alpha^{2}\right],
$$

where we choose $\alpha=1 / \sqrt{2}$ as in the classical case. The energy $E_{M}^{\text {quant }}$ exceeds both $E_{M}^{\mathrm{cl}}$ and the zero-point energy $E_{\mathrm{ZP}}=$ $\sum_{k=1}^{N} \hbar \omega_{k} / 2$. The quantum partition function reads $Z^{\text {quant }}=$ $\sum_{\mathbf{n}} e^{-\beta E_{\mathbf{n}}} \Theta\left(E_{M}^{\text {quant }}-E_{\mathbf{n}}\right)$, where $\Theta$ is the Heaviside function, illustrating an important difference with respect to gases of ground-state atoms. There, the truncation selects the trapped single-particle modes without constraining their populations, yielding a truncated Bose-Einstein distribution [50]. Instead, for Rydberg chains, the truncation involves the configuration energies $E_{\mathbf{n}}$. This prevents $Z^{\text {quant }}$ from factorizing and reflects the correlations between the trapped phonon modes, leading to a novel quasiequilibrium state that does not obey a truncated Bose-Einstein distribution.

We assume $E_{M}^{\text {quant }} \gg E_{\mathrm{ZP}}+\hbar \omega_{N}$, which is well satisfied for all parameters considered in this paper. Then, $E_{M}^{\text {quant }} \approx$ $E_{M}^{\mathrm{cl}}+E_{\mathrm{ZP}}$. For $k_{\mathrm{B}} T \gtrsim E_{M}^{\text {quant }} / N$, we evaluate the quantum en$\operatorname{ergy} U^{\text {quant }}(L, T)$ and entropy $S^{\text {quant }}(L, T)$ (see Appendix A 3) starting from Eq. (2), replacing $E_{M}^{\mathrm{cl}}$ by $E_{M}^{\text {quant }}$ and including the leading quantum correction, proportional to $\hbar^{2}$ (Ref. [61], Sec. 33). For $k_{\mathrm{B}} T<E_{M}^{\text {quant }} / N$, the energy and entropy reflect the nontruncated thermodynamics of a harmonic-oscillator chain. They overlap with $U^{\text {quant }}, S^{\text {quant }}$ for a range of values of $T$, yielding the full quantum thermodynamic functions (see Fig. 2).

\section{EVAPORATION}

We now describe the evaporation process. Initially, the chain is comprised of $N=N_{I}$ atoms in a trap of size $L^{(N)}=$ $L_{I}$, with the energy $U^{(N)}=U_{I}$. For all considered parameters, $U_{I} \gg E_{\mathrm{ZP}}$, signaling the classical regime, and $U_{I} \ll E_{M I}^{\mathrm{cl}}$, so that it is described by nontruncated thermodynamics. Thus,

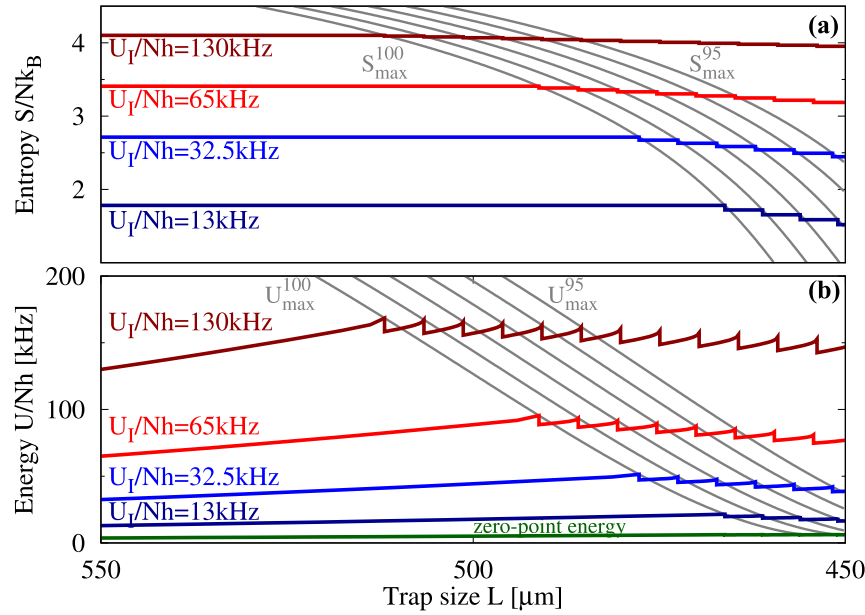

FIG. 3. The first few expulsions for a chain with $N_{I}=100$ atoms and $L_{I}=550 \mu \mathrm{m}$, in terms of (a) entropy $S / N$ and (b) energy $U / N$ per particle, for various initial energies. The maxima $S_{\max }^{(N)}(L) / N$ and $U_{\max }^{(N)}(L) / N$ are shown in gray. Each expulsion yields a discontinuity in both $S$ and $U$.

$U_{I} / N_{I}=k_{\mathrm{B}} T_{I}$ is the initial temperature. We adiabatically compress the chain by slowly decreasing $L$ (see Fig. 3). Hence, the entropy $S^{(N)}$ remains constant. Expelling an atom is irreversible, therefore $N$ also remains constant. However, $T$ and $U^{(N)}$ increase, whereas $U_{\max }^{(N)}(L)$ and $S_{\max }^{(\mathrm{N})}(L)$ decrease. The compression proceeds until the trap no longer accommodates the entropy, i.e., up to the trap size $L_{f}^{(N)}$ such that $S_{\max }^{(N)}\left(L_{f}^{(N)}\right)=$ $S^{(N)}$. This implies $T \rightarrow \infty$, hence $U_{f}^{(N)}=U_{\max }^{(N)}\left(L_{f}^{(N)}\right)$. At this point, the leftmost atom is expelled from the trap, its kinetic energy being the barrier height $V_{L}$. The $(N-1)$ remaining atoms thermalize to the new initial energy $U_{i}^{(N-1)}$, where

$$
U_{i}^{(N-1)}=U_{f}^{(N)}+V_{0}^{(N)}-V_{0}^{(N-1)}-V_{L}
$$

Here, $V_{0}^{(N)}$ and $V_{0}^{(N-1)}$ are the static equilibrium energies for $N$ and $(N-1)$ atoms in a trap of size $L_{f}^{(N)}$. Then, adiabatic compression resumes until the next expulsion.

The complete evaporation curve consists of a repeated sequence of these two steps. Figure 4 compares our classical (dark red) and quantum (red) predictions, down to the trap size $L_{F}=200 \mu \mathrm{m}$, where $E_{M}^{\text {quant }} \gtrsim E_{\mathrm{ZP}}+\hbar \omega_{N}$. The result of our classical model closely matches the classical-dynamics simulations reported in Ref. [27] (Fig. 14, phase II). Our quantum approach predicts that, starting from $N_{I}=100$ atoms, the final state with $N_{F}=40$ atoms obeys a Bose-Einstein distribution with $U_{F} /\left(N_{F} h\right)=7.0 \mathrm{kHz}$, slightly above the zero-point energy $E_{\mathrm{ZP}} /\left(N_{F} h\right)=5.9 \mathrm{kHz}$. The shown average energies account for the uncertainty $\Delta U_{I}=U_{I} / \sqrt{N_{I}}=h \times$ $6.5 \mathrm{kHz}$ on $U_{I}$, which washes out their jaggedness due to the expulsions (see Fig. 3 and Appendix A4). The final state is in the 1D regime if the radial confinement frequency $\omega_{\perp} /(2 \pi) \gg U_{F} /\left(N_{F} h\right)$. Smaller values of $\omega_{\perp}$ will lead to quasi-1D chains exhibiting the "zigzag" transition observed with ion chains [23,62] and in electronic systems [63]. 


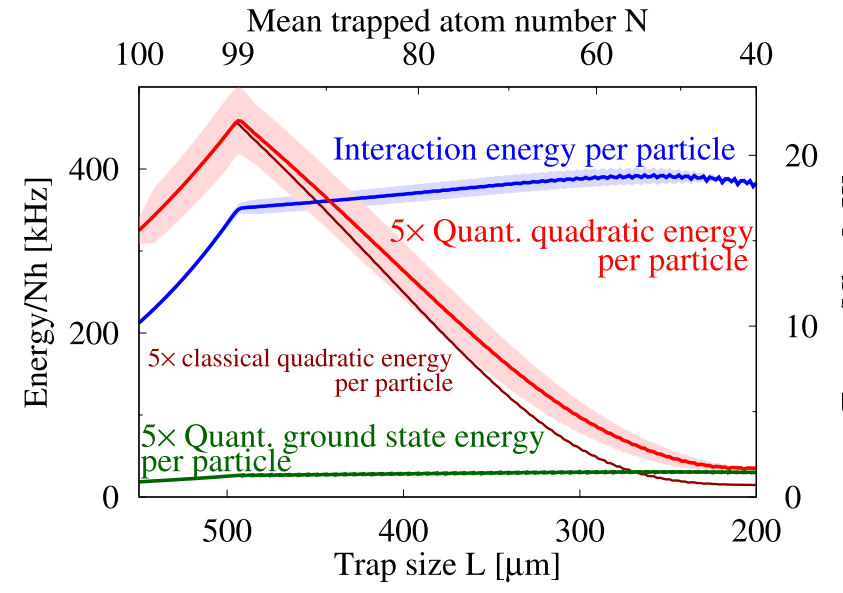

FIG. 4. Classical (dark red) and quantum (red) predictions for the mean quadratic energy, interaction (blue), and ground-state (green) energies per particle during the evaporation, starting from $N_{I}=100$, $L_{I}=550 \mu \mathrm{m}$, down to $N_{F}=40, L_{F}=200 \mu \mathrm{m}$. The shaded red and blue areas show the standard deviations on the quadratic and interaction energies. Energies are measured in $\mathrm{kHz}$, with $h \times 100 \mathrm{kHz} \sim$ $k_{\mathrm{B}} \times 5 \mu \mathrm{K}$.

\section{QUASIUNIVERSALITY FOR LONGER CHAINS}

Finally, we focus on long chains with $N_{I} \approx 1000$, keeping $l_{I}=L_{I} / N_{I}=5.5 \mu \mathrm{m}$. Then, the inhomogeneities near the trap edges are negligible, and both $S / N=s(l, T)$ and $U / N=$ $u(l, T)$ only depend on $l=L / N$ and $T$. The evaporation is conveniently described in terms of $l, s, u$, and the atom number fraction $n=N / N_{I}$. The evaporation curve consists of two parts (see Fig. 5). First, the initial compression at constant $N_{I}$ depends on $u_{I}=U_{I} / N_{I}$. The second part consists of all subsequent expulsions and compressions. The mean distance $l$ increases at each expulsion and decreases during each compression; on average, $l$ decreases. The quantities $s$ and $u$ always remain close to the universal curves $s_{\max }(l)=$ $S_{\max }(N, L) / N$ and $u_{\max }(l)=U_{\max }(N, L) / N$, respectively (see Appendix A 5). Their fluctuations, visible in the insets of Fig. 5 for $N_{I}=1000$ and $k_{\mathrm{B}} T_{I} / h=65 \mathrm{kHz}$, decrease with increasing $N_{I}$ for two reasons. First, the changes $\delta u$ and $\delta s$ in the energy and entropy per particle upon expelling an atom are decreasing functions of $N$. Second, larger $N_{I}$ lead to smaller $\Delta U_{I}=U_{I} / \sqrt{N_{I}}$, and hence to smaller uncertainties on $s$ and $u$. Quasiuniversality also applies to the fluctuations $\Delta u$ and $\Delta s$ on the energy and entropy (see Appendix A 5).

The fraction $n=N / N_{I}$ [Fig. 5(c)] is not universal (see Fig. 11 in the Appendix). For $N_{I}=1000, n$ reaches a stationary value $n_{F}$ as $u$ goes to $e_{\mathrm{ZP}}(l)=E_{\mathrm{ZP}}(l) / N$. The value $n_{F}\left(u_{I}\right)$ is a decreasing function of $u_{I}=U_{I} / N_{I}$. The curves in Fig. 5 are truncated at the minimum value $l=4.4 \mu \mathrm{m}$, where $E_{M}^{\text {quant }} \gtrsim E_{\mathrm{ZP}}+\hbar \omega_{N}$. Then, for $k_{\mathrm{B}} T_{I} / h=65 \mathrm{kHz}$, the chain comprises $N_{F}=764$ atoms with the energy $U_{F} /\left(N_{F} h\right)=$ $8.5 \mathrm{kHz}$, close to $E_{\mathrm{ZP}} /\left(N_{F} h\right)=6.6 \mathrm{kHz}$.

The final state of such a long chain is a crystal exhibiting true long-range order, with all spatial correlators $C_{n m}=$ $\left\langle\left(u_{n}-u_{m}\right)^{2}\right\rangle \ll l^{2}$ [see Fig. 6(c) in the Appendix]. This is only possible deep in the quantum regime, where thermal fluctuations are suppressed [14]. The crystalline order may be
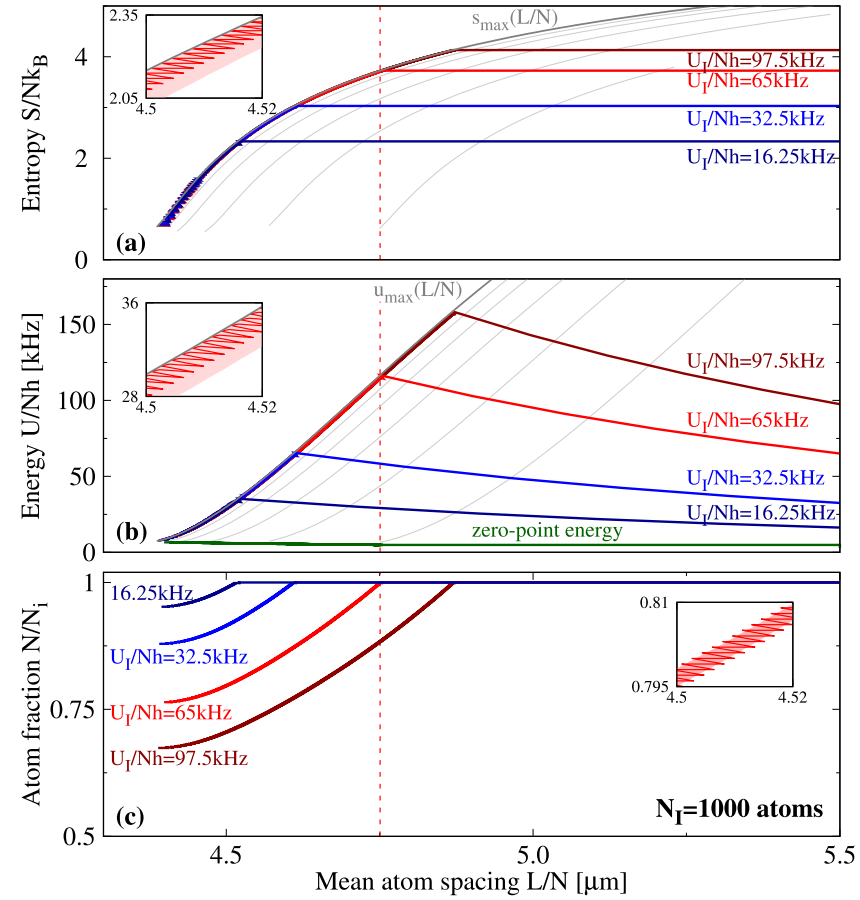

FIG. 5. Quasiuniversal evaporation of a chain with $N_{I}=1000$ and $l_{I}=5.5 \mu \mathrm{m}$, in terms of the mean (a) entropy and (b) energy per particle, for various initial energies. The thin gray lines show $S_{\max }(L / N) / N$ and $U_{\max }(L / N) / N$ for $N=50,100,200,400$, and 800 (from right to left), which converge toward $s_{\max }(l)$ and $u_{\max }(l)$ (thick gray lines). (c) Nonuniversal atom fraction $N / N_{I}$. The vertical red line shows the first expulsion for $U_{I} /\left(N_{I} h\right)=65 \mathrm{kHz}$. The insets zoom in on the same small fraction of the curves for $U_{I} /\left(N_{I} h\right)=$ $65 \mathrm{kHz}$, and show the jagged curves obtained before averaging; the shaded areas show the standard deviations.

fully characterized experimentally through microwave spectroscopy, revealing the regularity and fluctuations of the lattice parameter, combined with spatially resolved ground-state imaging [33,64].

\section{CONCLUSION AND OUTLOOK}

We have introduced a quantum thermodynamic model for the evaporative cooling of 1D Rydberg-atom chains [27]. Unlike the evaporative cooling of ground-state atoms, the final temperatures accessible with our scheme are not of the order of the barrier heights. Instead, they are determined by the maximum energy $u_{\max }(l)$ compatible with the trap. This reflects the many-body character of the evaporation scheme and leads to final temperatures that are radically lower than the barrier heights by three orders of magnitude. We have shown that, under realistic experimental conditions, this scheme yields large near-ground-state Rydberg crystal. The long-range spatial order of these $1 \mathrm{D}$ structures is a feature of the deep quantum regime. Our scheme will also apply to other interacting 1D systems such as polar molecules $[65,66]$. There, the nonzero-ranged interaction between the particles is provided by the dipole-dipole interaction, which scales with $1 / r^{3}$ and may be made purely repulsive in low-dimensional geometries [67]. 
The following directions warrant further investigation. (i) For higher initial temperatures or mean atom spacings, the initial state is a liquid and Eq. (1) does not hold, but our scheme will still drive the system toward its crystalline ground state. (ii) For longer chains, a prolonged evaporation going beyond the regime of Fig. 5 leads to $E_{M}^{\text {quant }} \lesssim E_{\mathrm{ZP}}+\hbar \omega_{N}$, in which case the calculation of the quantum thermodynamic functions is more involved. (iii) The timescale ensuring adiabaticity is set by the anharmonic processes neglected in Eq. (1). (iv) Our scheme is also applicable in two dimensions, where the expected ground state is a hexagonal crystal that we shall investigate both theoretically and experimentally.

\section{ACKNOWLEDGMENTS}

We acknowledge stimulating discussions with J. M. Raimond, P. Lecheminant, Y. Castin, T. Huillet, L. P. Pitaevskii, C. Sayrin, and G. V. Shlyapnikov.

\section{APPENDIX}

This Appendix provides complementary information on the following topics: (i) the applicability of the quadratic Hamiltonian; (ii) the anharmonic terms and their twofold role; (iii) the partition function and its numerical evaluation; (iv) the observability of the adiabatic plateaus with constant atom numbers; and (v) the quasiuniversal description for long chains and its limits.

\section{The quadratic Hamiltonian}

The Hamiltonian describing the harmonic vibrations of the atoms about their equilibrium positions $\left\{x_{n}^{0}\right\}$ [Eq. (1)] is applicable as soon as the chain exhibits local order. Indeed, in the chain bulk, the trapping potential is negligible and, within the nearest-neighbor approximation, the interaction energy of atom $n$ is $E_{n}^{I}=C_{6}\left[1 /\left(x_{n}-x_{n-1}\right)^{6}+1 /\left(x_{n}-x_{n+1}\right)^{6}\right]$. Here, $x_{n}=x_{n}^{0}+u_{n}$ is the position of atom $n$. Expanding $E_{n}^{I}$ to second order in the displacements $\left\{u_{n}\right\}$, and exploiting the near-translational invariance, we find that the harmonic approximation is valid if $\eta_{n}=21\left\langle\left(u_{n+1}-u_{n}\right)^{2}\right\rangle / l^{2}<1$, where $l=L / N$ is the mean interatomic distance and the average $\left\langle\left(u_{n+1}-u_{n}\right)^{2}\right\rangle$ is the spatial correlator between two neighboring atoms. For a thermal distribution, this condition reduces to $k_{\mathrm{B}} T<2 C_{6} / l^{6}$. Accounting for the trap and the truncated thermodynamics, we find this criterion to be well satisfied all along the evaporation [see Fig. 6(a)] for the long chain of Fig. 5.

The present criterion is less stringent than asking for the chain to be in a crystalline phase. This is especially true in one dimension, where thermal fluctuations quickly rule out long-range order [14]. For example, the long chain of Fig. 5 exhibits no long-range spatial correlations in its initial state $\left(N_{i}=1000, l_{i}=5.5 \mu \mathrm{m}, k_{\mathrm{B}} T_{i} / h=65 \mathrm{kHz}\right)$. This can be seen in Fig. 6(b): the correlator $\left\langle\left(u_{n}-u_{m}\right)^{2}\right\rangle / l^{2}>1$ for distant atoms. However, our scheme brings the chain close to its quantum ground state, which does exhibit long-range correlations $\left[\left\langle\left(u_{n}-u_{m}\right)^{2}\right\rangle / l^{2} \ll 1\right.$ for all $n$ and $m$; see Fig. 6(c)].

\section{Anharmonic effects}

The leading anharmonic contribution to the Hamiltonian follow from the third- and fourth-order terms in the displacements $\left\{u_{n}\right\}$. For gases, they yield two-body collisions that are essentially instantaneous. By contrast, for Rydberg chains, they generate many-body correlations over the characteristic time $\tau_{\text {propag }}$ for propagation along the chain, set by the sound velocity. They are mostly due to interactions and occur in the chain bulk, where their probability does not depend on position (see Fig. 7). They are much less probable near the edges, where the trapping potential leads to larger distances between the static equilibrium positions of the atoms.

The role of these anharmonic processes is twofold. First, they are responsible for thermalization and ergodicity on a timescale involving $\tau_{\text {propag. }}$. Second, they set the (longer) timescale ensuring the adiabaticity of the compression between two atomic expulsions. The classical-dynamics simulations reported in Ref. [27] have shown that, for the shorter chain of Fig. $3\left(N_{i}=100\right)$, compression rates of the order of $40 \mu \mathrm{m} / \mathrm{ms}$ are adequate. The optimal compression rate will be investigated elsewhere.

For gases, anharmonic processes directly drive the atomic expulsions, which immediately follow two-atom collisions during which one atom has acquired enough energy. Their relation to expulsions is more involved for Rydberg chains. If the trap size is such that an expulsion is expected $(T \rightarrow \infty)$, ergodicity causes the system to explore various configurations until the leftmost atom is expelled with the energy $V_{L}$. If no expulsion is expected ( $T$ finite), the compression of the trap causes an increase in energy due to the atoms on the edges of
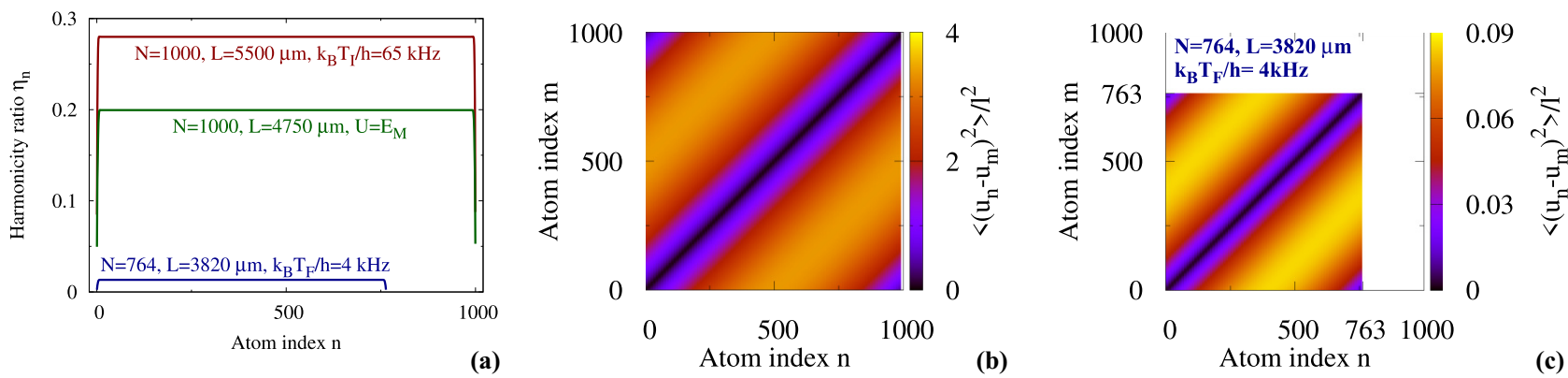

FIG. 6. (a) Harmonicity ratio $\eta_{n}$ for the long chain of Fig. 5, at the beginning of the evaporation (red), just before the first expulsion (green), and at the end of the evaporation (blue). (b) and (c) Spatial correlator $\left\langle\left(u_{n}-u_{m}\right)^{2}\right\rangle / l^{2}$ at the beginning $[(\mathrm{b}), N=1000, L=5500 \mu \mathrm{m}]$ and the end [(c), $N=764, L=3820 \mu \mathrm{m}]$ of the evaporation, in units of $l=L / N$. 


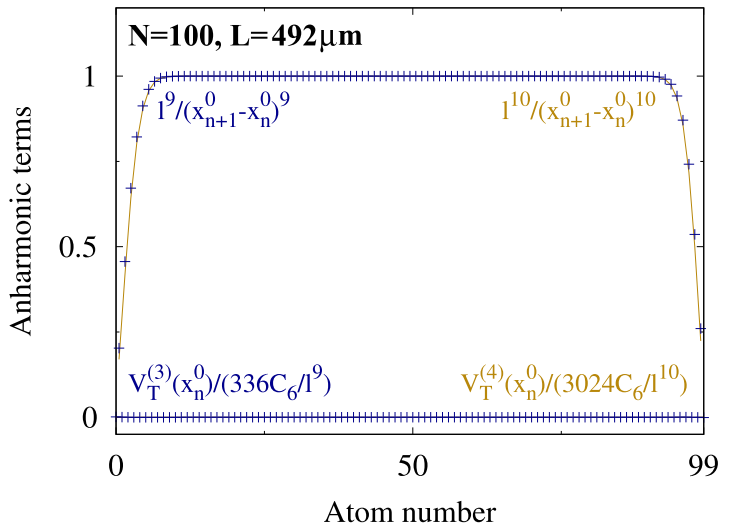

FIG. 7. Third- (blue crosses) and fourth-order (golden lines) anharmonic amplitudes from the interaction energy (nonzero amplitudes) and the trapping potential (negligible amplitudes), calculated for the shorter chain of Fig. 3, just before the first expulsion. They are expressed in units of their bulk values, namely $56 C_{6} / l^{9}$ and $126 C_{6} / l^{10}$.

the chain being set in motion toward the bulk. Expelling the leftmost atom before thermalization has taken place (i.e., with an energy $>V_{L}$ ) is likely to involve a two-atom collision at the open end of the trap. There, anharmonic terms are strongly suppressed (see Fig. 7), so that these higher-energy expulsions are rare. Instead, the energy increase is most often mediated, through harmonic vibrations, to the chain bulk where thermalization occurs. The rare cases in which the leftmost atom is expelled are not captured by our thermodynamic model. However, they are not a hindrance as long as their rate remains small: instead, they speed up the evaporation process with respect to our thermodynamic prediction. The presence of a single open end (the left end in Fig. 1) is favorable for two reasons: (i) it leads to longer propagation times, and hence more efficient thermalization; and (ii) it helps reduce the rate of nonthermalized expulsions.

\section{The partition function}

\section{a. Normalized lower incomplete $\gamma$ function}

The thermodynamics of the (classical or quantum) truncated Boltzmann distribution involve the normalized lower incomplete $\gamma$ function $P(a, z)$, defined as [59]

$$
P(a, z)=\frac{\gamma(a, z)}{\Gamma(a)}=\frac{1}{\Gamma(a)} \int_{0}^{a} d t e^{-t} t^{z-1} .
$$

For given values of the trap size $L$ and atom number $N$, the classical partition function $Z^{\mathrm{cl}}$ is proportional to $P\left(N, \beta E_{M}\right) / \beta^{N}$. Hence, $a$ is of the order of $N$, whereas $z=$ $\beta E_{M}$ is the ratio of the threshold energy to the temperature. For a given $a$, the function $P(a, z)$ resembles a step function [see Fig. 8(a)] which is equal to 0 for small $z$ (representing the truncation for large $T$ ) and to 1 for large $z$ (truncation plays no role for small $T$ ). The smooth transition occurs for $z \approx a$, so that truncation plays a role for $k_{\mathrm{B}} T / E_{M} \gtrsim 1 / a$. The parameter $a=3$ for a gas in a truncated 3D harmonic trap [51], whereas for Rydberg chains $a \approx N$ ranges from 40 to 1000 . Hence, Rydberg chains are affected by the truncation starting from much lower temperatures than gases are.

\section{b. Quantum partition function}

For a given $L$, and assuming $E_{M}^{\text {quant }} \gg E_{\mathrm{ZP}}+\hbar \omega_{N}$, we evaluate the quantum partition function $Z^{\text {quant }}$ for $k_{\mathrm{B}} T \gtrsim$ $E_{M}^{\text {quant }} / N$ using Eq. (2) in the main text, replacing $E_{M}^{\mathrm{cl}}$ by $E_{M}^{\text {quant }}$. We go beyond the quasiclassical integral expression and include the leading-order quantum correction, proportional to $\hbar^{2}$ (Ref. [61], Sec. 33). Hence, we write $Z^{\text {quant }}=$ $Z^{\mathrm{cl}}\left(1+\left\langle\hbar^{2} \chi_{2}\right\rangle\right)$, where the correction $\left\langle\hbar^{2} \chi_{2}\right\rangle$ is expressed in terms of the moments $\left\langle x_{k}^{2}\right\rangle_{\mathrm{cl}},\left\langle p_{k}^{2}\right\rangle_{\mathrm{cl}}$, and $\left\langle x_{k}^{2} p_{k}^{2}\right\rangle_{\mathrm{cl}}$ of $Z_{\mathrm{cl}}$. We find

$$
\begin{aligned}
& \left\langle\hbar^{2} \chi_{2}\right\rangle E_{M}^{2} / \sum_{k=1}^{N}\left(\hbar \omega_{k}\right)^{2} \\
& \quad=\frac{z^{2}}{24}\left(-1+[3 z-5(N+1)] \frac{z^{N} e^{-z}}{\Gamma(N+2)} \frac{1}{P(N, z)}\right),
\end{aligned}
$$

with $z=\beta E_{M}$. For $k_{\mathrm{B}} T<E_{M} / N$, we use the quantum partition function $Z_{0}=\prod_{k=1}^{N}\left[\operatorname{csch}\left(\beta \hbar \omega_{k} / 2\right) / 2\right]$ of a nontruncated chain. The functions $U^{\text {quant }}$ and $S^{\text {quant }}$ overlap with those extracted from $Z_{0}$ for a range of values of $k_{\mathrm{B}} T$, thus yielding the full quantum thermodynamic functions. The classical and quantum predictions for $U$ and $S$ are compared in Fig. 8. At the beginning of the evaporation [panel (b)], they only differ over a narrow range of temperatures near $T=0$; the
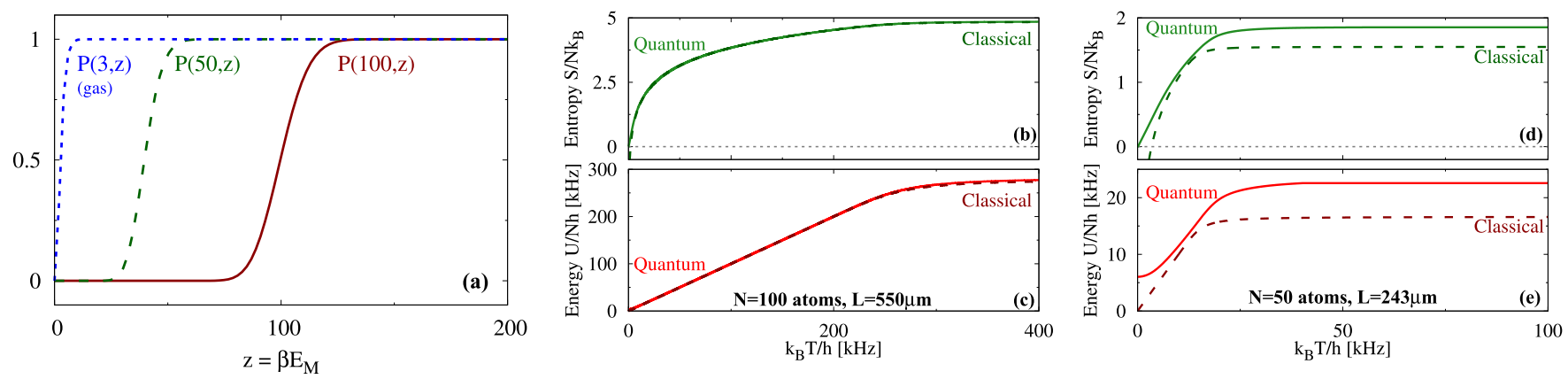

FIG. 8. (a) Normalized incomplete $\gamma$ function $P(a, z)$ as a function of $z=\beta E_{M}$ for $a=3,50$, and 100. (b) and (c) $s=S / N$ and $u=U / N$ as a function of $T$ for 100 atoms in a trap of size $L=550 \mu \mathrm{m}$ (beginning of the evaporation in Fig. 4 of the main text). (d) and (e) $s$ and $u$ for 50 atoms with $L=243 \mu \mathrm{m}$ (close to the end of the evaporation in Fig. 4). 


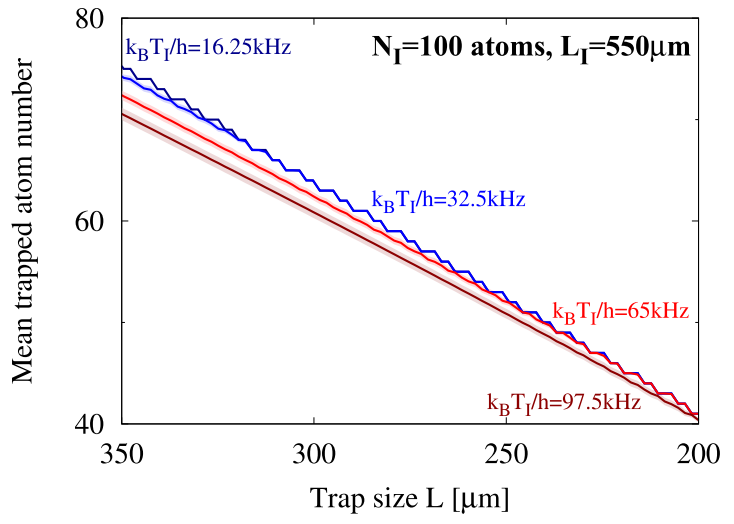

FIG. 9. Mean trapped atom number as a function of the trap size $L$ for the short chain initially comprising $N_{I}=100$ atoms with $L_{I}=$ $550 \mu \mathrm{m}$ for various initial temperatures. The red curve, calculated for $k_{\mathrm{B}} T_{I}=65 \mathrm{kHz}$, corresponds to Fig. 4 of the main text. The discrete steps on the atom number become visible at the end of the evaporation; for lower initial temperatures, they are well resolved earlier on. The shaded areas show the mean standard deviation due to the initial fluctuations $\Delta U_{I}=U_{I} / \sqrt{N_{I}}$ on the quadratic energy.

difference is more striking near the end of the evaporation [panel (c)].

\section{c. Numerical evaluation}

The evaluation of $U(L, T)$ and $S(L, T)$ involves calculating $P(a, z)$ for $40 \leqslant a \leqslant 1000$. To capture the steep variation of these functions for $z \sim a$, we resort to arbitrary-precision numerics using the BOOST.MULTIPRECISION C++ library [68].

\section{Constant atom number plateaus}

Between two atomic expulsions, the chain undergoes an adiabatic compression during which $N$ remains constant (see
Fig. 3). For $k_{\mathrm{B}} T_{I} / h \sim 65 \mathrm{kHz}$, these constant $-N$ plateaus are smoothed out for most of the evaporation because of the uncertainty $\Delta U_{I}=U_{I} / \sqrt{N_{I}}$ on the initial energy. Indeed, it reflects on the entropy as $\Delta S_{I}=\Delta U_{I} / T_{I}$, and leads to sizable fluctuations $\Delta S^{(N)}$ during most of the evaporation. These yield the uncertainty $\Delta L_{f}^{(N)}=\Delta S^{(N)} / S_{\max }^{(N) \prime}\left(L_{f}\right)$ on the trap size $L_{f}^{(N)}$ at which the atom $N$ is expelled.

For shorter chains, the constant- $N$ plateaus become well resolved at the end of the evaporation, as the chain approaches its ground state. For Fig. 4 and $k_{\mathrm{B}} T_{I} / h=65 \mathrm{kHz}$, these plateaus are visible when the remaining trapped atom number $N \lesssim 45$ (see Fig. 9), in agreement with the classical-dynamics results of Ref. [27]. The plateaus are resolved earlier on for lower initial temperatures and later on for higher ones.

\section{Quasiuniversality}

We now focus on longer chains with $N \sim 1000$ and $l \sim$ $5 \mu \mathrm{m}$. Then, the quadratic energy $U(N, L, T)=N u(l, T)$, the entropy $S(N, L, T)=N s(l, T)$, their maxima $U_{\max }(N, L)=$ $N u_{\max }(l)$ and $S_{\max }(N, L)=N s_{\max }(l)$, and the zero-point en$\operatorname{ergy} E_{\mathrm{ZP}}(N, l)=N e_{\mathrm{ZP}}(l)$ are all extensive.

\section{a. Energy and entropy}

We consider two consecutive adiabatic plateaus corresponding to $N$ and $N-1$ trapped atoms. Equation (5) provides the initial energy per particle $u_{i}^{(N-1)}=U_{i}^{(N-1)} /(N-1)$ for the second plateau in terms of its final value for the first one, $u_{f}^{(N)}=U_{f}^{(N)} / N$, and the mean distance $l_{f}^{(N)}=L_{f}^{(N)} / N$ :

$$
u_{i}^{(N-1)}=u_{f}^{(N)}+\left(u_{f}^{(N)}+7 C_{6} / l_{f}^{(N) 6}-V_{L}\right) / N .
$$

Hence, starting from the first atomic expulsion, $u$ remains close to the universal curve $u=u_{\max }(l)$, within small deviations that decrease like $1 / N$. Furthermore, the entropies $s^{(N)}=$ $s_{\max }\left(l_{f}^{(N)}\right)$, which are constant during each plateau, all lie near
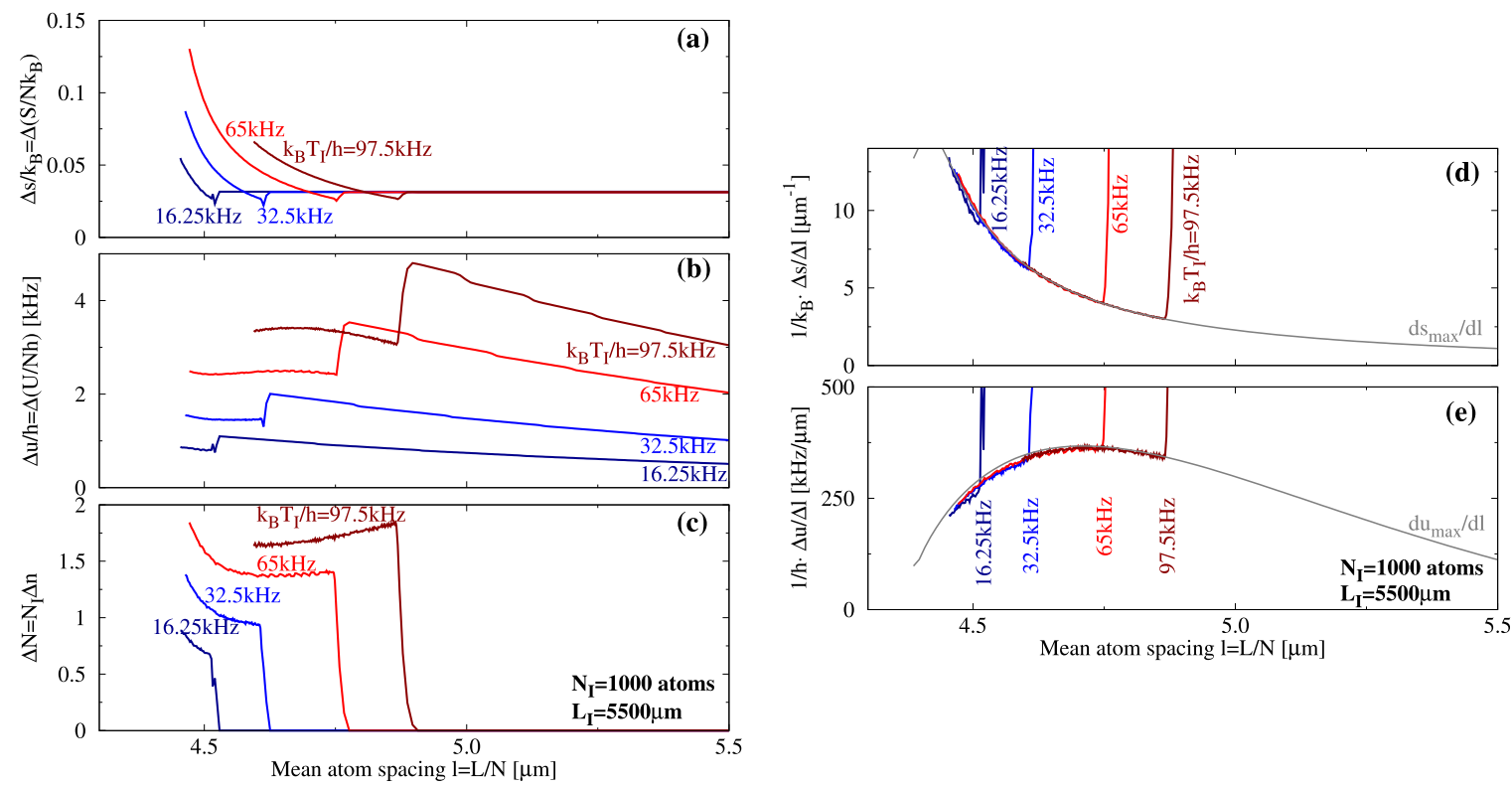

FIG. 10. Standard deviations (a) $\Delta s$, (b) $\Delta u$, and (c) $\Delta n$ on the entropy per particle $s=S / N$, the energy per particle $u=U / N$, and the remaining atom fraction $n=N / N_{I}$, for the long chain of Fig. 5 in the main text. The ratios (d) $\Delta s / \Delta l$ and (e) $\Delta u / \Delta l$, with $\Delta l=(l / n) \Delta n$, closely follow the derivatives $d s_{\max } / d l$ and $d u_{\max } / d l$ starting from the first expulsion. 

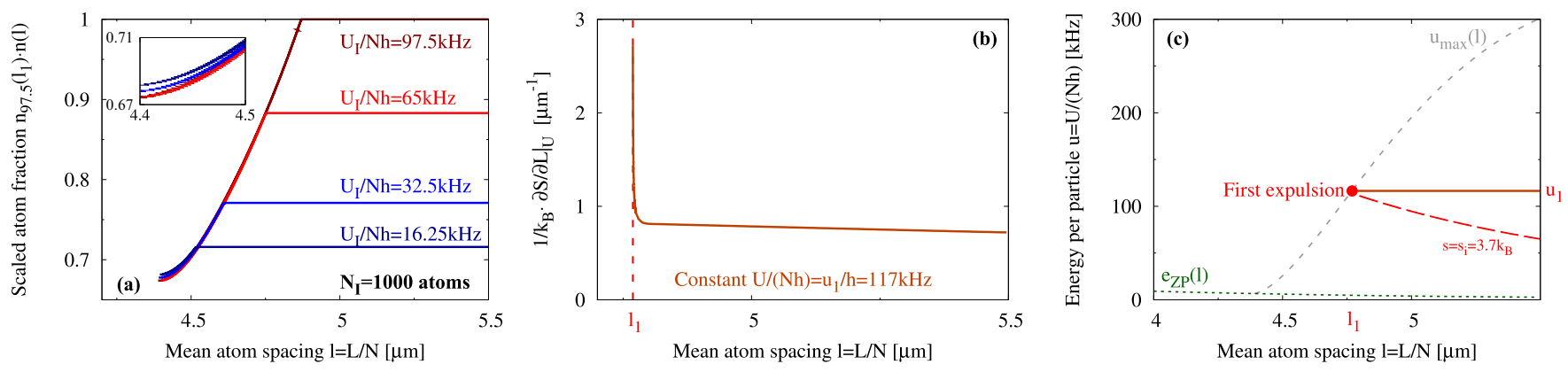

FIG. 11. (a) Scaled atom fraction $n=N / N_{I}$ for $N_{I}=1000$ and various $u_{I}$, showing an approximate scaling whose breakdown is visible in the inset. (b) The derivative $\partial s /\left.\partial l\right|_{u}$ calculated along the horizontal dark red path in panel (c). This path crosses the curve $u=u_{\max }(l)$ (dashed gray line) at the first expulsion point $\left(l_{1}, u_{1}\right)$ for $u_{I} / h=65 \mathrm{kHz}$. The dotted-dashed red line shows the isentropic curve followed up to the first expulsion.

the universal curve $s=s_{\max }(l)$. Both of these properties are illustrated in Fig. 5 for various $u_{I}=U_{I} / N_{I}$, which set the mean atomic distance $l_{1}$ at which the first expulsion occurs.

\section{b. Fluctuations}

The quasiuniversality of the evaporation constrains the fluctuations $\Delta u$ and $\Delta s$ on the energy and entropy per particle to follow those on the atomic distance, $\Delta l$. Neglecting the small deviations from the universal curves $u=u_{\max }(l)$ and $s=s_{\max }(l)$, they satisfy $\Delta u / \Delta l=u_{\max }^{\prime}(l)$ and $\Delta s / \Delta l=$ $s_{\text {max }}^{\prime}(l)$ (see Fig. 10).

The constraint on $\Delta u / \Delta l$ has an important consequence. As $l$ decreases, $u_{\max }(l)$ tends toward $e_{\mathrm{ZP}}(l)$ [see Fig. 5(b)]. Hence, the derivative $u_{\max }^{\prime}(l)$ goes to zero. The fluctuations $\Delta u$ do not vanish, therefore $\Delta l$ increases and so does $\Delta n=$ $(n / l) \Delta l$ [see Fig. 10(a)]. Thus, as long as the quasiuniversal regime holds, the constant- $N$ plateaus will be poorly resolved. If the evaporation proceeds further, it will eventually drive the system out of the universal regime. Then, we expect to recover the short-chain behavior described in Appendix A 4. For the chain considered in Figs. 5 and 10, this occurs beyond the validity range of our assumption $E_{M}^{\text {quant }} \gg E_{\mathrm{ZP}}+\hbar \omega_{N}$, and will be investigated elsewhere.

\section{c. Nonuniversality of $N / N_{I}$}

The entropy per particle $s(l, u)$ may be seen as a function of $l$ and $u$. The derivative $\partial s /\left.\partial u\right|_{l}=1 / T$ goes to zero on the curve $u=u_{\max }(l)$, which is reached for $T \rightarrow \infty$. However, our numerical results show that $\partial s /\left.\partial l\right|_{u}$ diverges along the curve $u=u_{\max }(l)$ [see Fig. 11(b)]. Therefore, $s(l, u)$ may not be linearized near this curve, and the entropy difference $s^{(N-1)}-s^{(N)}=s\left(l_{i}^{(N-1)}, u_{i}^{(N-1)}\right)-s\left(l_{f}^{(N)}, u_{f}^{(N)}\right)$ goes to zero slower than $1 / N$. This rules out any exact universal behavior for the atom number fraction $n=N / N_{I}$. However, the deviation from universality is small. For a given $N_{I}$, we consider two initial energies $u_{I 1}<u_{I 2}$, and we compare the curves $n_{u_{I 1}}(l)$ and $n_{u_{I 2}}(l)$ for $l<l_{1}$, where $l_{1}$ is the mean atom spacing leading to the first expulsion for $u_{I 1}$. Our numerical results show that these two curves nearly satisfy the scaling relation, which would have been exact had $\partial s /\left.\partial l\right|_{u}$ not been divergent, namely $n_{u_{I 2}}\left(l_{1}\right) n_{u_{I 1}}(l) \approx n_{u_{I 2}}(l)$ [see Fig. $11(\mathrm{a})$, whose inset highlights the breakdown of this scaling behavior].

The divergence of $\partial s /\left.\partial l\right|_{u}=p / T$ along the curve $u=$ $u_{\text {max }}(l)$ signals that the pressure $p$ goes to infinity faster than $T$ does. This starkly contrasts with the behavior of the ideal gas, where $p / T=n k_{\mathrm{B}}$ is finite, its constant value being set by the particle density $n$.
[1] E. Wigner, Phys. Rev. 46, 1002 (1934).

[2] C. C. Grimes and G. Adams, Phys. Rev. Lett. 42, 795 (1979).

[3] J. G. Bohnet, B. C. Sawyer, J. W. Britton, M. L. Wall, A. M. Rey, M. Foss-Feig, and J. J. Bollinger, Science 352, 1297 (2016).

[4] E. Jordan, K. A. Gilmore, A. Shankar, A. Safavi-Naini, J. G. Bohnet, M. J. Holland, and J. J. Bollinger, Phys. Rev. Lett. 122, 053603 (2019).

[5] R. A. Guyer, Solid State Phys. 23, 413 (1970).

[6] E. B. Sonin, Rev. Mod. Phys. 59, 87 (1987).

[7] I. Coddington, P. Engels, V. Schweikhard, and E. A. Cornell, Phys. Rev. Lett. 91, 100402 (2003).

[8] A. Haziot, X. Rojas, A. D. Fefferman, J. R. Beamish, and S. Balibar, Phys. Rev. Lett. 110, 035301 (2013).

[9] J. Li, J. Lee, W. Huang, S. Burchevsky, B. Shteynas, F. C. Top, A. O. Jamison, and W. Ketterle, Nature (London) 543, 91 (2017).
[10] J. Léonard, A. Morales, P. Zupancic, T. Esslinger, and T. Donner, Nature (London) 543, 87 (2017).

[11] L. Tanzi, E. Lucioni, F. Famà, J. Catani, A. Fioretti, C. Gabbanini, R. N. Bisset, L. Santos, and G. Modugno, Phys. Rev. Lett. 122, 130405 (2019).

[12] F. Böttcher, J. N. Schmidt, M. Wenzel, J. Hertkorn, M. Guo, T. Langen, and T. Pfau, Phys. Rev. X 9, 011051 (2019).

[13] L. Chomaz, D. Petter, P. Ilzhöfer, G. Natale, A. Trautmann, C. Politi, G. Durastante, R. M. W. van Bijnen, A. Patscheider, M. Sohmen, M. J. Mark, and F. Ferlaino, Phys. Rev. X 9, 021012 (2019).

[14] N. D. Mermin, Phys. Rev. 176, 250 (1968).

[15] D. Vu and S. Das Sarma, Phys. Rev. B 101, 125113 (2020).

[16] P. Jurcevic, B. P. Lanyon, P. Hauke, C. Hempel, P. Zoller, R. Blatt, and C. F. Roos, Nature (London) 511, 202 (2014). 
[17] P. Richerme, Z. Gong, A. Lee, C. Senko, J. Smith, M. Foss-Feig, S. Michalakis, A. V. Gorshkov, and C. Monroe, Nature (London) 511, 198 (2014).

[18] C. Senko, J. Smith, P. Richerme, A. Lee, W. C. Campbell, and C. Monroe, Science 345, 430 (2014).

[19] R. Lechner, C. Maier, C. Hempel, P. Jurcevic, B. P. Lanyon, T. Monz, M. Brownnutt, R. Blatt, and C. F. Roos, Phys. Rev. A 93, 053401 (2016).

[20] V. V. Deshpande and M. Bockrath, Nature Phys. 4, 314 (2008).

[21] I. Shapir, A. Hamo, S. Pecker, C. P. Moca, O. Legeza, G. Zarand, and S. Ilani, Science 364, 870 (2019).

[22] S. Balibar, J. Beamish, A. Fefferman, A. Haziot, X. Rojas, and F. Souris, C. R. Phys. 17, 264 (2016).

[23] H. L. Partner, R. Nigmatullin, T. Burgermeister, J. Keller, K. Pyka, M. B. Plenio, A. Retzker, W. H. Zurek, A. del Campo, and T. E. Mehlstäubler, Physica B 460, 114 (2015).

[24] D. Kleppner, M. G. Littman, and M. L. Zimmerman, Sci. Am. 244, 130 (1981).

[25] M. Saffman, T. G. Walker, and K. Mølmer, Rev. Mod. Phys. 82, 2313 (2010)

[26] Y. Zeng, P. Xu, X. He, Y. Liu, M. Liu, J. Wang, D. J. Papoular, G. V. Shlyapnikov, and M. Zhan, Phys. Rev. Lett. 119, 160502 (2017).

[27] T. L. Nguyen, J. M. Raimond, C. Sayrin, R. Cortiñas, T. Cantat-Moltrecht, F. Assemat, I. Dotsenko, S. Gleyzes, S. Haroche, G. Roux, T. Jolicoeur, and M. Brune, Phys. Rev. X 8, 011032 (2018).

[28] H. Weimer, M. Müller, I. Lesanovsky, P. Zoller, and H. P. Büchler, Nat. Phys. 6, 382 (2010).

[29] T. Pohl, E. Demler, and M. D. Lukin, Phys. Rev. Lett. 104, 043002 (2010).

[30] P. Schauss, M. Cheneau, M. Endres, T. Fukuhura, S. Hild, A. Omran, T. Pohl, C. Gross, S. Kuhr, and I. Bloch, Nature (London) 491, 87 (2012).

[31] P. Schauss, J. Zeiher, T. Fukuhara, S. Hild, M. Cheneau, T. Macri, T. Pohl, I. Bloch, and C. Gross, Science 347, 1455 (2015).

[32] H. Bernien, S. Schwartz, A. Keesling, H. Levine, A. Omran, H. Pichler, S. Choi, A. S. Zibrov, M. Endres, M. Greiner, V. Vuletic, and M. D. Lukin, Nature (London) 551, 579 (2017).

[33] D. Barredo, V. Lienhard, S. de Léséleuc, T. Lahaye, and A. Browaeys, Nature (London) 561, 79 (2018).

[34] H. Labuhn, D. Barredo, S. Ravets, S. de Leseleuc, T. Macri, T. Lahaye, and A. Browaeys, Nature (London) 534, 667 (2016).

[35] S. de Léséleuc, V. Lienhard, P. Scholl, D. Barredo, S. Weber, N. Lang, H. P. Büchler, T. Lahaye, and A. Browaeys, Science 365, 775 (2019).

[36] G. Pupillo, A. Micheli, M. Boninsegni, I. Lesanovsky, and P. Zoller, Phys. Rev. Lett. 104, 223002 (2010).

[37] Y. Jau, A. M. Hankin, T. Keating, I. H. Deutsch, and G. W. Biedermann, Nat. Phys. 12, 71 (2016).

[38] J. Zeiher, R. van Bijnen, P. Schauss, S. Hild, J. Choi, T. Pohl, I. Bloch, and C. Gross, Nat. Phys. 12, 1095 (2016).

[39] R. Löw, H. Weimer, J. Nipper, J. B. Balewski, B. Butscher, H. P. Büchler, and T. Pfau, J. Phys. B 45, 113001 (2012).
[40] A. W. Glaetzle, M. Dalmonte, R. Nath, I. Rousochatzakis, R. Moessner, and P. Zoller, Phys. Rev. X 4, 041037 (2014).

[41] H. Weimer, R. Löw, T. Pfau, and H. P. Büchler, Phys. Rev. Lett. 101, 250601 (2008).

[42] R. Löw, H. Weimer, U. Krohn, R. Heidemann, V. Bendkowsky, B. Butscher, H. P. Büchler, and T. Pfau, Phys. Rev. A 80, 033422 (2009).

[43] R. G. Hulet and D. Kleppner, Phys. Rev. Lett. 51, 1430 (1983).

[44] D. A. Anderson, A. Schwarzkopf, R. E. Sapiro, and G. Raithel, Phys. Rev. A 88, 031401(R) (2013).

[45] V. Zhelyazkova and S. D. Hogan, Phys. Rev. A 94, 023415 (2016).

[46] A. Signoles, E. K. Dietsche, A. Facon, D. Grosso, S. Haroche, J. M. Raimond, M. Brune, and S. Gleyzes, Phys. Rev. Lett. 118 253603 (2017).

[47] D. Kleppner, Phys. Rev. Lett. 47, 233 (1981).

[48] R. G. Hulet, E. S. Hilfer, and D. Kleppner, Phys. Rev. Lett. 55, 2137 (1985).

[49] R. G. Cortiñas, M. Favier, B. Ravon, P. Méhaignerie, Y. Machu, J. M. Raimond, C. Sayrin, and M. Brune, Phys. Rev. Lett. 124, 123201 (2020).

[50] M. Yamashita, M. Koashi, and N. Imoto, Phys. Rev. A 59, 2243 (1999).

[51] O. J. Luiten, M. W. Reynolds, and J. T. M. Walraven, Phys. Rev. A 53, 381 (1996).

[52] W. D. Johnston, Jr. and J. G. King, Phys. Rev. Lett. 16, 1191 (1966).

[53] P. W. Anderson, Phys. Lett. A 29, 563 (1969).

[54] F. Dalfovo, A. Fracchetti, A. Lastri, L. Pitaevskii, and S. Stringari, Phys. Rev. Lett. 75, 2510 (1995).

[55] D. J. Papoular, L. P. Pitaevskii, and S. Stringari, Phys. Rev. A 94, 023622 (2016)

[56] S. K. Dutta, J. R. Guest, D. Feldbaum, A. Walz-Flannigan, and G. Raithel, Phys. Rev. Lett. 85, 5551 (2000).

[57] L. Allen, S. M. Barnett, and M. J. Padgett, Optical Angular Momentum (Institute of Physics, Bristol, UK, 2003).

[58] M. Brune and J. M. Raimond (private communication).

[59] DLMF, NIST Digital Library of Mathematical Functions, http: //dlmf.nist.gov/ (2018).

[60] J. T. M. Walraven, Quantum Dynamics of Simple Systems (IOP, Bristol, UK, 1996).

[61] L. D. Landau and E. M. Lifshitz, Statistical Physics, Part 1, 3rd ed. (Elsevier, Amsterdam, 1980).

[62] G. Birkl, S. Kassner, and H. Walther, Nature (London) 357, 310 (1992).

[63] H. Ikegami, H. Akimoto, D. G. Rees, and K. Kono, Phys. Rev. Lett. 109, 236802 (2012).

[64] D. Barredo, S. de Léséleuc, V. Lienhard, T. Lahaye, and A. Browaeys, Science 354, 1021 (2016).

[65] L. De Marco, G. Valtolina, K. Matsuda, W. G. Tobias, J. P. Covey, and J. Ye, Science 363, 853 (2019).

[66] L. Anderegg, L. W. Cheuk, Y. Bao, S. Burchesky, W. Ketterle, K. Ni, and J. M. Doyle, Science 365, 1156 (2019).

[67] M. A. Baranov, M. Dalmonte, G. Pupillo, and P. Zoller, Chem. Rev. 112, 5012 (2012).

[68] BOOST.MuLTIPRECISION library, https://www.boost.org/doc/ libs/1_70_0/libs/multiprecision/doc/html/index.html (2019). 